\title{
Giant cell arteritis in a patient with fever of unknown origin
}

\author{
Timur Selcuk Akpinar ${ }^{1}$, Busra Alpaslan ${ }^{1}$, Raim Iliaz $^{2}$, Goknur E. Isik $^{3}$, Bulent Saka $^{1}$ \\ ${ }^{1}$ Department of Internal Medicine, Istanbul Medical Faculty, Istanbul University, Istanbul, Turkey \\ ${ }^{2}$ Department of Internal Medicine, Division of Geriatrics, Istanbul Medical Faculty, Istanbul University, Istanbul, Turkey \\ ${ }^{3}$ Department of Nuclear Medicine, Istanbul Medical Faculty, Istanbul University, Istanbul, Turkey \\ Email: doktortimur@gmail.com, busra@gmail.com, goknur@yahoo.com, bulentsaka@gmail.com, raimiliaz@gmail.com
}

Received 5 April 2013; revised 5 May 2013; accepted 13 May 2013

Copyright (C 2013 Timur Selcuk Akpinar et al. This is an open access article distributed under the Creative Commons Attribution License, which permits unrestricted use, distribution, and reproduction in any medium, provided the original work is properly cited.

\begin{abstract}
Fever of unknown (FUO) origin challenges clinical condition. The causes of FUO are diverse: possible etiologies include infectious diseases, chronic granulomatous diseases, malignity, connective tissue disorders and vasculitis. Giant cell arteritis which affects the aorta and its main branches, contributes up to $17 \%$ of cases with fever of unknown origin above age 50. There is increasing evidence for a role for $18 \mathrm{~F}$ FDG PET/CT in the diagnosis of vasculitis and 18FFDG PET/CT is a useful technic for evaluation of the FUO. Clinical studies show that FDG-PET is a useful diagnostic aid in obscure cases of fever of unknown origin. We report here a case of a 54-year-old woman who presented in to our clinic with FUO due to giant cell vasculitis.
\end{abstract}

Keywords: Fever of Unknown Origin; Vasculitis; PET/CT

\section{INTRODUCTION}

Fever of unknown origin is defined as a temperature higher than $38.3^{\circ} \mathrm{C}$ that lasts for more than three weeks with no obvious source despite one week of inpatient investigation. The causes of FUO are diverse; the spectrum of FUO etiology may include more than 200 diseases [1]. Possible etiologies include infectious diseases, chronic granulomatous diseases, malignancy, connective tissue disorders and vasculitis. Vasculitis is defined by the presence of inflammatory leukocytes in vessel walls with reactive damage to surrounding tissues. It is usually classified according to the size of the vessel involved. Giant cell arteritis which affects the aorta and its main branches, contributes up to $17 \%$ of cases with fever of unknown origin above age 50. We present a case of giant cell arteritis diagnosed by PET-CT in a patient with fever of unknown origin.

\section{CASE}

A 54-year-old woman presented with persistent high fever. Her right leg was amputated above the knee three years ago due to extensive vessel and nerve injury caused by a meniscus repair. She was diagnosed with pulmonary embolism during her hospital stay and a vena cava filter was inserted. Two months ago she started complaining of fatigue, loss of appetite and high fever. Following initial evaluation at the clinic, she was admitted to the ward with fever of unknown origin. On physical examination she looked pale, no sign of infection was observed on the stump. Her blood pressure was within normal limits and pulses were palpable bilaterally without murmur. Laboratory findings were consistent with iron deficiency anemia (Hb: $8 \mathrm{gr} / \mathrm{dl}, \mathrm{MCV}: 83 \mathrm{fL}$, iron: $13 \mu \mathrm{g} / \mathrm{dl}$, TIBC: $180 \mu \mathrm{g} / \mathrm{dl}$, Ferritin: $660 \mathrm{ng} / \mathrm{ml}$ ), high sensitive CRP was $220 \mathrm{mg} / \mathrm{L}$ and sedimentation rate $140 \mathrm{~mm} / \mathrm{hr}$. Viral serology and romatologic serologic parameters were negative. Blood and urine cultures taken during fever were sterile. Peripheral blood smear was consistent with iron deficiency, but gastroscopy and colonoscopy revealed no major pathologies. Ultrasound of the surgical area of the knee did not show any signs of infection or abscess. Echocardiography revealed moderate-severe pericardial effusion. Thorax CT showed circular thickening of the ascending and descending thoracic aorta and the aortic arch, in addition to pericardial effusion and bilateral mild pleural effusion. PET-CT was then performed and revealed diffuse hypermetabolism on the walls of bilateral common carotids, bilateral subclavian arteries, ascending aorta, aortic arch, descending aorta, abdominal aorta and bilateral femoral arteries (Figure 1). The patient was diagnosed as giant cell arteritis. Systemic methotrexate 


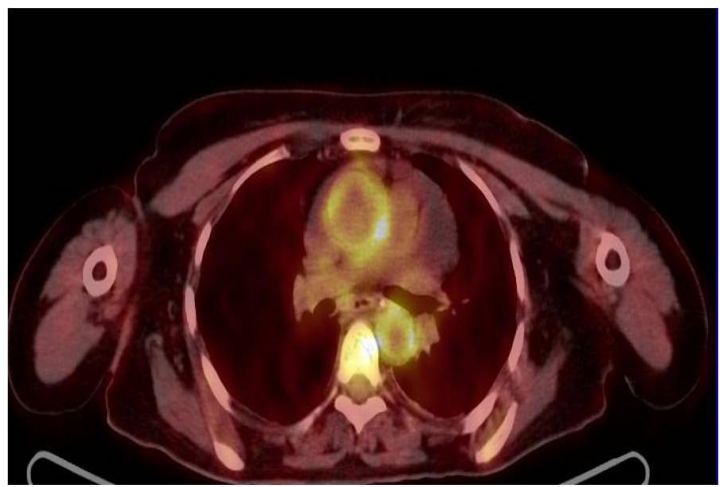

Figure 1. PET/CT image showing giant cell arteritis.

and steroid therapy was started. After this treatment the patient's fever was relieved and acute phase reactants (CRP, sedimentation, ferritin) decreased to normal levels.

\section{DISCUSSION}

Fluoro-deoxy-glucose (FDG) is a glucose metabolite proved useful for tracing metabolically active cells. The diagnostic value of Positron emission tomography (PET) depends on the increased FDG uptaken by malignant and inflammatory lesions. There is increasing evidence for a role for 18F-FDG PET/CT in the diagnosis of vasculitis. In the study with the total number of nine patients only one of the nine patients with large vessel vasculitis presenting with FUO was diagnosed incorrectly after performing PET/CT scans, indicating that PET/CT is highly sensitive [2]. PET is a non-invasive and high speed method which shows the anatomical distribution of the vessel wall inflammation. It is especially valuable in the diagnosis of vasculitis with non specific symptoms. Clinical studies show that FDG-PET is a useful diagnostic aid in obscure cases of fever of unknown origin. 18FFDG PET or PET/CT is a sensitive techniques for the evaluation of FUO. However, it is still not a routine procedure in the workup of FUO due to its high cost and limited availability.

\section{REFERENCES}

[1] Gaeta, G.B., Fusco, F.M. and Nardiello, S. (2006) Fever of unknown origin: A systematic review of the literature for 1995-2004. Nuclear Medicine Communications, 27, 205-211. doi:10.1097/00006231-200603000-00002

[2] Amberger, C.C., Dittmann, H., Overkamp, D., et al. (2005) Large vessel vasculitis as cause of fever of unknown origin (FUO) or systemic inflammation. Diagnosis using 18-F-fluor-2-deoxy-D-glucose positron emission to mography ((18) F-FDG-PET). Zeitschrift für Rheumatologie, 64, 32-39. doi:10.1007/s00393-005-0639-2 